\title{
Structural studies of a novel ubiquitin-modifying enzyme, SdeA using various tools
}

Leehyeon Kim (1), Do Hoon Kwon (2) \& Hyun Kyu Song (3)

(1) Department of Life Sciences, Korea University, 145 Anam-ro, Seongbuk-gu, Seoul 02841, Korea, leehyeon@korea.ac.kr

(2) Department of Life Sciences, Korea University, 145 Anam-ro, Seongbuk-gu, Seoul 02841, Korea, dohoon.kwon@duke.edu

(3) Department of Life Sciences, Korea University, 145 Anam-ro, Seongbuk-gu, Seoul 02841, Korea, hksong@korea.ac.kr

Post-translational attachment of ubiquitin ( $\mathrm{Ub}$ ) is one of the most common protein modifications and is involved in a number of key cellular processes in eukaryotes. Conventional ubiquitylation occurs through an ATP-dependent three-enzyme cascade (E1, E2, and E3) that mediates the covalent conjugation of the $\mathrm{C}$-terminus of ubiquitin to a lysine on the substrate. SdeA, which belongs to the SidE effector family of Legionella pneumophila, can transfer ubiquitin to endoplasmic reticulum-associated Rab-family GTPases in a manner independent of E1 and E2 enzymes [1]. The novel ubiquitin-modifying enzyme SdeA utilizes NAD+ as a cofactor to attach ubiquitin to a serine residue of the substrate $[2,3]$.

Here, we determined the overall structure of the $180 \mathrm{kDa}$ full-length SdeA and the spatial orientation of the $\mathrm{N}$-terminal deubiquitylase (DUB), phosphodiesterase (PDE), mono ADP-ribosyltransferase (mART), and C-terminal coiled-coil (CC) domains using small angle X-ray scattering (SAXS) technique. The full-length shape was checked through negative staining. Besides, crystal structures of the mART-C domain of SdeA was determined in free form and in complex with NAD+ at high resolution. The sub-domains of mono ADP-ribosyltransferase termed mART-N and mART-C were characterized to elucidate the coupled enzymatic reaction of NAD+ hydrolysis and ADP-ribosylation of ubiquitin. These results provide insight into the unusual ubiquitylation mechanism of SdeA and expand our knowledge of ubiquitin biology.

\section{References}

[1] Qui, J. et al. (2016). Nature, 533, 120-124

[2] Bhogaraju, S. et al. (2016). Cell, 167, 1636-1649

[3] Bhogaraju, S. \& Dikic, I. (2016). Nature, 533, 43-44 